\title{
Power Transformer Partial Discharge Signal De-noising Based on Variational Mode Decomposition
}

\author{
Lei Zhao ${ }^{1, a}$, Chungeng $\mathrm{Li}^{1,2, b}$, Yongli Zhu ${ }^{1, \mathrm{c}}, \mathrm{Xu} \mathrm{Chen}^{1}$, Xiaohong Guo ${ }^{1}$ and \\ Yanfeng $\mathrm{Gao}^{1}$ \\ ${ }^{1}$ North China Electric Power University, Baoding 071003 \\ ${ }^{2}$ Hengshui Electric Power Supply Company, China \\ azhaoleizozelar@163.com, bhg_lcg@sohu.com, cyonglipw@163.com
}

Keywords: transformer, partial discharge, VMD, signal de-noising.

\begin{abstract}
Partial discharge (PD) signals is one of the key means to judge the transformer fault, how to effectively remove interference of PD in the field is the key in PD monitoring. Based on the Hilbert transform, this paper proposes a new algorithm of signal decomposition of Variational Mode Decomposition (VMD) by using the de-noising in transformer PD signal, VMD can decompose signal into several modes, and each of them is compact around a center pulsation. The simulation results show that the proposed method can well remove the narrow-band periodic interference and white noise, and keep the partial discharge signal, verify the validity of VMD.
\end{abstract}

\section{Introduction}

Power transformer is one of the important equipments of the power system, its reliable operation has great significance for safety, economic operation of power system. The reliability of the power transformer mainly depends on the insulation condition, and one of the main causes of transformer insulation aging and damage is PD, so transformer PD on-line monitoring has high theoretical and practical value to improve power system reliability and economy. One of the main problems of PD on-line monitoring is the interference, including continuous and periodic narrowband interference and white noise which are the most serious [1].

Recently wavelet transform (WT) has been used to PD de-noising, noise and PD signals can be separated into different coefficients by WT. By applying thresholds to the coefficients, the noise will be discarded and the PD signals will be kept for reconstructing the de-noised PD signals.[2] Empirical Mode Decomposition (EMD), an adaptive technique, decomposes a signal into a series of IMFs, which have different frequency scales. But the EMD method has the mixed mode phenomena, so that it's hard to extract PD signals from narrowband interference [3].

In this paper, a new method for transformer PD signal de-noising is proposed, the proposed VMD method can decompose the input signal into a series of sub-signals (modes), and each mode are tightly around a center pulsation. VMD can filter the white noise out adaptively, and can decompose the rest into a series of modes. Extract the mode containing PD signals then strip the PD signals from noisy signals and achieve a better result of signal de-noising.

\section{Brief review of VMD theory and algorithm}

VMD theory. VMD process is variational problem solving, and involves three important concepts: the classic Wiener filter, Hilbert transform and frequency mixing.

Variational problem. We assume each mode to be mostly compact around a center pulsation, and describe variational problem as seeking $\mathrm{k}$ modes $u_{k}$, minimizing the estimated bandwidth of each mode, and its constraint is the sum of each mode is equal to the input signal $f$. Specific configuration steps are as follows:

1) For each mode $u_{k}$, compute the analytic signal by means of the Hilbert transform in order to obtain a unilateral frequency spectrum: 


$$
\left(\delta(t)+\frac{j}{\pi t}\right) * u_{k}(t)
$$

2) For each mode, shift the mode's frequency spectrum to "baseband", by mixing with an exponential tuned $e^{-j \omega_{k} t}$ to the respective estimated center frequency:

$$
\left[\left(\delta(t)+\frac{j}{\pi t}\right) * u_{k}(t)\right] e^{-j \omega_{k} t}
$$

3) Compute the squared $L^{2}$-norm of the gradient of the demodulated signal, estimate the bandwidth, the resulting constrained variational problem is the following:

$$
\begin{array}{r}
\min _{\left\{u_{k}\right\},\left\{w_{k}\right\}}\left\{\sum_{k}\left\|\partial_{t}\left[\left(\delta(t)+\frac{j}{\pi t}\right) * u_{k}(t)\right] e^{-j \omega_{k} t}\right\|^{2}\right\} \\
\text { s.t. } \sum_{k} u_{k}=f
\end{array}
$$

where $\left\{u_{k}\right\}:=\left\{u_{1}, \ldots, u_{K}\right\},\left\{\omega_{k}\right\}:=\left\{\omega_{1}, \ldots, \omega_{K}\right\}, \sum_{k}:=\sum_{k-1}^{K}$.

Solve the variational problem.

1) Make use of both a quadratic penalty term and Lagrangian multiplier, in order to render the problem unconstrained .The quadratic penalty can encourage reconstruction fidelity, typically in the presence of additive i.i.d Gaussian noise. Lagrangian multipliers are a common way of enforcing constrains strictly. The augmented Lagrangian $\mathcal{L}$ as follows:

$$
\begin{aligned}
\mathcal{L}\left(\left\{u_{k}\right\},\left\{\omega_{k}\right\}, \lambda\right) & :=\alpha \sum_{k}\left\|\partial_{t}\left[\left(\delta(t)+\frac{j}{\pi t}\right) * u_{k}(t)\right] e^{-j \omega_{k} t}\right\|^{2} \\
& +\left\|f(t)-\sum_{k} u_{k}(t)\right\|_{2}^{2}+\left\langle\lambda(t), f(t)-\sum_{k} u_{k}(t)\right\rangle .
\end{aligned}
$$

2) VMD makes use of alternate direction method of multipliers to search the saddle point of the augmented Lagrangian $\mathcal{L}$ by updating $u_{k}^{n+1}, \omega_{k}^{n+1}, \lambda^{n+1}$ alternately.

\section{Complete optimization of VMD}

1) Initialize $\left\{\hat{u}_{k}^{1}\right\},\left\{\omega_{k}^{1}\right\}, \hat{\lambda}^{1}, n$.

2) Update $u_{k}, \omega_{k}, \lambda: \hat{\lambda}^{n+1}(\omega) \leftarrow \hat{\lambda}^{n}(\omega)+\tau\left(\hat{f}(\omega)-\sum_{k} \hat{u}_{k}{ }^{n+1}(\omega)\right)$

3) For $e>0$, convergence: $\sum_{k}\left\|\hat{u}_{k}^{n+1}-\hat{u}_{k}^{n}\right\|_{2}^{2} /\left\|\hat{u}_{k}^{n}\right\|_{2}^{2}<e$, otherwise , return step 2) [4].

Furthermore, the algorithm is very simple. First, each mode updates directly in the frequency domain. Second, as the power spectrum of each mode, the center frequency is re-estimated for circularly iteration.

\section{VMD on simulated signals}

Simulated PD signals. PD signals are high frequency signals, however the PD signals can be distorted and attenuated when they propagate from their respective discharge locations to PD detectors. Transformer partial discharge signals tested in field are generally damped oscillation signal, such signals can be used in the form of a single exponential decay oscillation and double 
exponential decaying oscillation forms [5]:

$$
\begin{aligned}
& f(t)=A e^{-\left(t-t_{0}\right) / \tau} \sin \left(2 \pi f_{c}\left(t-t_{0}\right)\right) \\
& f(t)=A\left(e^{-1.3\left(t-t_{0}\right) / \tau}-e^{-2.2\left(t-t_{0}\right) / \tau}\right) \sin \left(2 \pi f_{c}\left(t-t_{0}\right)\right), t>t_{0}
\end{aligned}
$$

where $A$ is the signal amplitude, $\tau$ is the duration of $f(t), t_{0}$ is the start time of the discharge pulse, $f_{c}$ is the oscillatory frequency of $f(t)$. Periodic noise and white noise which are very common in PD data acquisition were injected to the original PD signal [6]. Sampling frequency of simulated signals is $10 \mathrm{MHz}$, and the data length is 2000. The frequency of periodic noise $p(t)$ contains $100,350,550 \mathrm{kHz}$ and $1 \mathrm{MHz}$, and white noise $n(t)$ is normal distribution with mean value 0 and variance value 0.001 . Computer simulation of detected transformer partial discharge signals as:

$$
s(t)=f(t)+p(t)+n(t)
$$

Partial discharge simulation waveform parameters given in the paper as shown in the Table 1, the corresponding time domain waveform as shown in Fig. 1. Table 1 Partial discharge model parameters

\begin{tabular}{ccccc}
\hline & $A(V)$ & $t_{0}(\mu \mathrm{s})$ & $f_{c}(\mathrm{kHz})$ & $\tau(\mu \mathrm{s})$ \\
\hline PD1 & 0.01 & 30 & 200 & 5 \\
\hline PD2 & 0.03 & 120 & 200 & 5 \\
\hline
\end{tabular}

To evaluate the de-noising performance of the proposed method, three measures including the signal-to-noise ratio before and after interference suppression $S N R 1$ and $S N R 2$, noise suppression ratio NNR [7] and mean square error ( MSE ) [8] were adopted. They are defined as:

$$
\begin{aligned}
& S N R 1=10 \lg \left(P_{S 1} / P_{U 1}\right)=10 \lg \left(P_{S 1} / \sigma_{1}^{2}\right) \\
& S N R 2=10 \lg \left(P_{S 2} / P_{U 2}\right)=10 \lg \left(P_{S 2} / \sigma_{2}^{2}\right) \\
& N R R=10\left(\lg \sigma_{1}^{2}-\lg \sigma_{2}^{2}\right) \\
& M S E=\frac{\sum_{i=1}^{N}(Y(i)-X(i))^{2}}{\operatorname{length}(Y(i))} \times 100 \%
\end{aligned}
$$

where $P_{S 1}, P_{S 2}$ are the power values of real PD signal and processed detected PD signal. $P_{U 1}$, $P_{U 2}$ are power values of noise before and after processing. $\sigma_{1}, \sigma_{2}$ are noise deviations. $Y(i)$, $X(i)$ are real PD signal and de-noising PD signal respectively. length $(Y(i))$ is the data length.

Fig. 2 shows that PD signals almost drowned in the noise, Fig. 3 shows the noisy signal spectrum. Before using VMD, we should predefine the number of modes $k$, both overbinning and underbinning have predictable impact on the nature of detected modes. In this paper, $k$ is 5 , Fig. 4 shows the 5 decomposed modes. And the second mode (Mode 2, shown in Fig. 5) contains the most PD signal, the rest modes are the periodic noise signals, the white noise, filtered out after decomposition does not appear in any mode. As PD signal is non-stationary signal, the spectral 
bands of modes can change more drastically over time, and will globally overlap, the de-noised PD signal contains some small periodic interference.

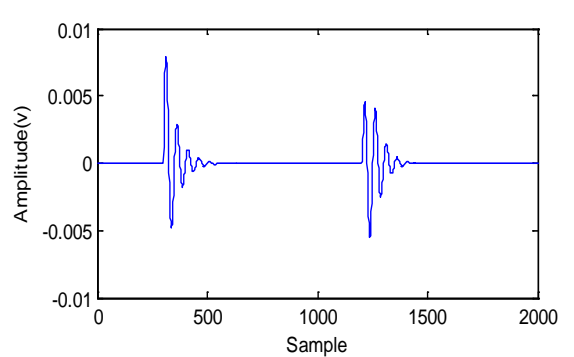

Fig.1 Original signal

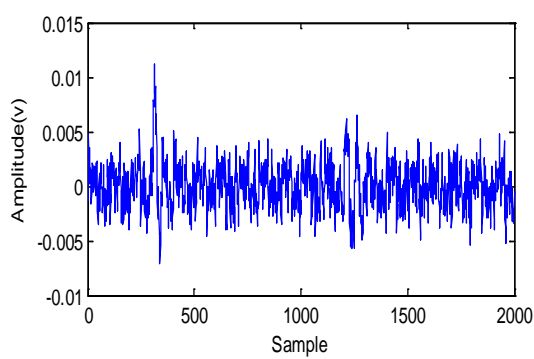

Fig.2 Noisy signal

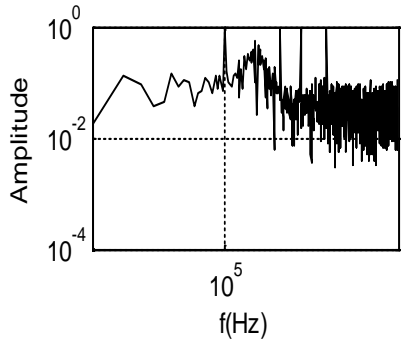

Fig.3 Input signal spectrum

The Table 2 shows the performance evaluation of the proposed method, and the results show that VMD can filter out periodic noise and white noise effectively and can preserve PD signal well.

Table 2 Performance of VMD

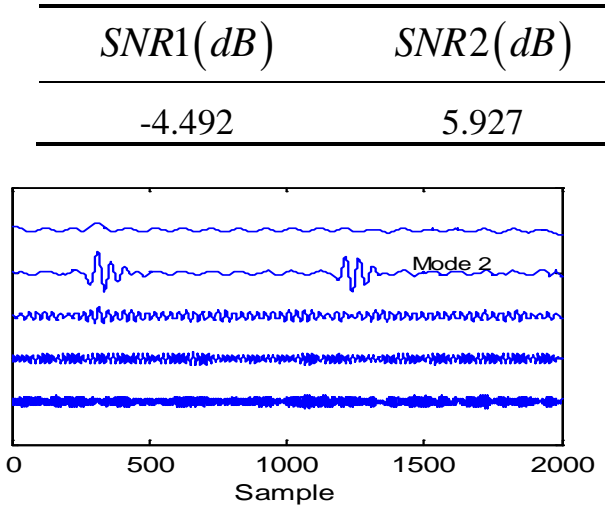

Fig.4 Decomposed modes

$N N R(d B) \quad M S E(\%)$

$0.0021 \%$

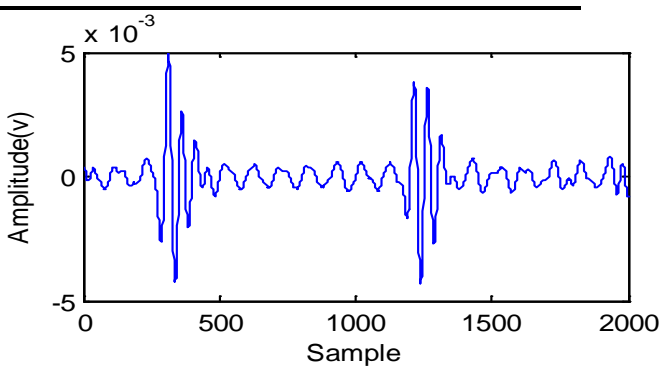

Fig.5 Mode 2

\section{Summary}

This paper proposes a transformer PD signal de-noising method based on VMD, through VMD, the noisy signal is decomposed into several modes, each of which is around a center pulsation, then the PD signal is separated from the noisy signal, and thus achieving a satisfactory de-noising effect.

\section{References}

[1] Yunpeng Liu, Fangcheng Lv, Chengrong Li and Yanqing Li: Proceedings of the CSEE, Vol.24(2004),p.169.

[2] Yizhi Mao, Song Li and Zhanqiang Li: TRANSFORMER, Vol. 48(2011),p.47-50.

[3] Linpeng Yao, Wendong Zheng, Qian Yong, Yongping Du, et al: Power System Protection and Control, Vol. 39(2011),p.133-139.

[4] Konstantin Dragomiretskiy and Dominique Zosso: IEEE TRANSACTIONS ON SIGNAL PROCESSING, Vol. X(2013),p.1-14.

[5] Jeffery C. Chan, Hui Ma, Tapan K. Saha and Chandima Ekanayake: IEEE Transactions on Dielectrics and Electrical Insulation, Vol. 21, No. 1,p.296; February 2014.

[6] Ju Tang, Caixin Sun: High Voltage Engineering, Vol.28(2002),p.8-10.

[7] Yizhi Mao, Wei Wang, Dongmei Li and Ziliang Yang: TRANSFORMER,Vol.51(2014),p.34.

[8] R.V. Maheswari, P. Subburaj, B. Vigneshwaran, and M. Willjuice Iruthayarajan: J Electr Eng Technol Vol.9, No.2, p. 695-706, 2014. 